\title{
Infants who develop eczema at 12 months have a deficient $T$ regulatory cell response to microbial stimuli at the time of birth
}

\begin{abstract}
Rationale $\mathrm{T}$ Regulatory (Treg) cells and may play an essential role in regulating early immune development and shaping the immune response toward a proallergic or tolerant status. Proinflammatory cytokines may be associated with tolerance induction. Methods Cord blood mononuclear cells of 72 neonates at high risk allergy whose mothers participated in a prenatal probiotic eczema prevention study (PEPS) were cultured with TLR2 ligandslipoteichoic acid (LTA) and heat-killed LGG (HKL), a TLR4 ligand - lipopolysaccharide (LPS), ovalbumin (OVA), anti-CD3 or media for 48 hours. Numbers of Treg and production of Treg and inflammatory cytokines were compared between children who developed eczema at any time during the first year of life $(n=24)$ and those who did not $(n=48)$. Results Infants who developed eczema had lower percentages of FoxP3hiCD25hi CD4+ Treg cells after LTA $(\mathrm{p}=0.009)$ and HKL $(\mathrm{p}=0.04)$ stimulations, and lower IL-6 production following HKL induction $(\mathrm{p}=0.03)$ than those without eczema. No differences in the percentages of Foxp3hiCD25hi Treg following stimulation with OVA, LPS, or anti-CD3. There was a negative correlation between the SCORAD score and the percentage of Treg cells induced by LTA $(r=-0.48, p=0.0002)$. Conclusions Children who develop allergic disease in the first year of life have deficient Treg and inflammatory responses to microbial stimuli from the time of birth. A reduced ability to generate Treg cells and IL-6 in response to microbial stimuli could be a contributing factor to the failure of normal tolerance development in infancy, and this warrants further investigation.
\end{abstract}

Keyword: Eczema; Infants; T Regulatory (Treg); Microbial stimuli; Allergic disease 